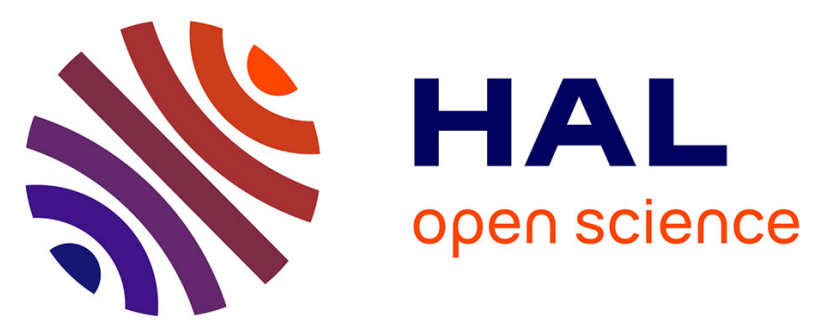

\title{
Single Fiber Transport in a Fracture Slit: Influence of the Wall Roughness and of the Fiber Flexibility
}

Maria Veronica d'Angelo, Benoît Semin, Guillemette Picard, Martin Poitzsch, Jean-Pierre Hulin, Harold Auradou

\section{> To cite this version:}

Maria Veronica d'Angelo, Benoît Semin, Guillemette Picard, Martin Poitzsch, Jean-Pierre Hulin, et al.. Single Fiber Transport in a Fracture Slit: Influence of the Wall Roughness and of the Fiber Flexibility. Transport in Porous Media, 2010, 84 (2), pp.389-408. 10.1007/s11242-009-9507-x . hal00442661

\section{HAL Id: hal-00442661 \\ https://hal.science/hal-00442661}

Submitted on 22 Dec 2009

HAL is a multi-disciplinary open access archive for the deposit and dissemination of scientific research documents, whether they are published or not. The documents may come from teaching and research institutions in France or abroad, or from public or private research centers.
L'archive ouverte pluridisciplinaire HAL, est destinée au dépôt et à la diffusion de documents scientifiques de niveau recherche, publiés ou non, émanant des établissements d'enseignement et de recherche français ou étrangers, des laboratoires publics ou privés. 


\title{
Single fiber transport in a fracture slit: influence of the wall roughness and of the fiber flexibility.
}

\author{
M. V. D'Angelo · B. Semin · G. Picard · M. E. \\ Poitzsch · J.P. Hulin · H. Auradou
}

Received: date / Accepted: date

\begin{abstract}
The transport of fibers by a fluid flow is investigated in transparent channels modelling rock fractures: the experiments use flexible polyester thread (mean diameter $280 \mu \mathrm{m}$ ) and water or a water-polymer solution. For a channel with smooth parallel walls and a mean aperture $\bar{a}=0.65 \mathrm{~mm}$, both fiber segments of length $\ell=20-150 \mathrm{~mm}$ and "continuous" fibers longer than the channel length have been used: in both cases, the velocity of the fibers and its variation with distance could be accounted for while neglecting friction with the walls. For rough self-affine walls and a continuous gradient of the local mean aperture transverse to the flow, transport of the fibers by a water flow is only possible in the region of larger aperture $(\bar{a} \gtrsim 1.1 \mathrm{~mm})$ and is of "stop and go" type at low velocities. With the polymer solution, the fibers move faster and more continuously in high aperture regions and their interaction with the walls is reduced; fiber transport becomes also possible in narrower regions where irreversible pinning occurred for water. In a third rough model with parallel walls and a low mean aperture $\bar{a}=0.65 \mathrm{~mm}$, fiber transport is only possible with the water-polymer solution. The dynamics of fiber deformations and entanglement during pinning-depinning events and permanent pinning is analyzed.
\end{abstract}

Keywords fracture $\cdot$ fibers $\cdot$ roughness $\cdot$ flow $\cdot$ buckling $\cdot$ pinning $\cdot$ polymer

PACS $46.32 .+\mathrm{x} \cdot 47.50 .-\mathrm{d} \cdot 47.56 .+\mathrm{r} \cdot 47.15 . \mathrm{gp}$

M.V. D'Angelo $\cdot$ B. Semin · J. P. Hulin · H. Auradou

Univ Pierre et Marie Curie-Paris 6, Univ Paris-Sud, CNRS, Lab. FAST, Bâtiment 502, Campus Paris-Sud, Orsay, $F-91405$ France.

Tel.: 33 (0) 169158084

Fax: 33 (0) 169158060

E-mail: vdangelo@fo.uba.fr,semin@fast.u-psud.fr,hulin@fast.u-psud.fr,auradou@fast.u-psud.fr.

Present address: M. V. D’Angelo

Grupo de Medios Porosos, Facultad de Ingenieria, Paseo Colon 850, 1063, Buenos-Aires, Argentina.

G. Picard · M. E. Poitzsch

Schlumberger-Doll Research, 1 Hampshire Street, Cambridge, MA 02139 U.S.A.

E-mail: gpicard@slb.com,poitzsch1@slb.com. 


\section{Introduction}

This paper deals with the transport by a fluid flow of long, flexible fibers in a single fracture bounded by two rough walls with a small spacing between them. In addition to its fundamental interest, this problem has potential applications such as the transport and filtration of biological macromolecules like actin or DNA [Lagomarsino et al. 2004] or the transport of nanotubes or of industrial polymers in small channels. At a much larger scale, optical fibers might also be used as in-situ probes for measuring the temperature (or other parameters) of water flows in natural rocks [Selker et al. 2006].

The transport of fibers by a fluid flow has been widely studied but for flow geometries and fiber properties differing generally from the present work. Many studies deal with suspensions of short rigid fibers encountered in the manufacturing of composite and/or reinforced materials: a great deal of experiments [Herzhaft and Guazzelli 1999] and numerical simulations [Fan et al. 1998, Butler and Shaqfeh 2002, Saintillan et al. 2005] characterize, for instance, their sedimentation. The orientation of the fibers in the flow of such suspensions inside slit-like channels has also been investigated [Yasuda et al. 2002, Chiba et al. 1988].

Suspensions of flexible fibers are equally frequent in the paper pulp industry [Stockie 1998] and in biotechnology. Recent numerical simulations analyze the viscosity and normal stresses in unbounded shear flows of these suspensions as well as their dependence on the deformations and motion of the fibers [Yamamoto and Matsuoka 1995, Joung 2001, Tornberg and Shelley 2004].

The case of single fibers has been often studied in $2 D$ shear flow configurations with the fiber in the shear plane. Since the pioneering work of Jeffery [Jeffery 1923] demonstrating periodic motions for rigid fibers, these studies have been extended to flexible fibers [Forgacs and Mason 1959, Hinch 1976, Yamamoto and Matsuoka 1993, Ning and Melrose 1999]. While most papers dealt with unbounded shear flows, the influence of a wall perpendicular to the shear plane was studied experimentally by [Moses 2001] and numerically by [Skjetne et al. 1997]: a "pole vaulting" effect was demonstrated for fibers close enough to the walls.

Large amplitude deformations of long filaments or flagellae are also encountered in microorganisms and account for their propulsion: both cases of isolated microorganisms [Taylor 1951, Purcell 1997, Lowe 2003] and of several interacting ones [Llopis et al. 2008] have been studied. In another study, the motion of a flexible fiber in an infinite fluid is, in contrast, driven by a body force and deformations of the fiber occur [Lagomarsino et al. 2005].

In the above studies, the flow field was either unbounded or bound by walls separated by a distance large compared to the length of the fibers; in our experiments, this length is, in contrast, much larger than the distance of the walls. In this case, most deformations of the fiber and variations of its mean orientation take place in the mean plane of the fracture. The deformations in the direction of the aperture are strongly constrained and may lead to strong interactions with the walls. Then a key issue is whether the fibers can be transported by the flow to the desired location or whether blockage occurs and/or the flow channels get clogged: such blockage effects will be particularly important for rough walls.

Here, we use long flexible fibers and study experimentally their transport by a fluid flow in transparent model fractures with either smooth or rough walls. While the former is a reference case, the geometry of the latter is close to that of encountered in natural rocks and in materials used in civil, environmental and petroleum engineering. A first set of experiments ([D'Angelo et al. 2009]) demonstrated important qualitative differences between the propagation of fibers in models with smooth and rough walls (velocity fluctuations are much larger in the second case). Also, replacing water by a polymer solution as the flowing fluid led to a faster and more continuous motion of the fibers. 
Here, the dynamics of the fibers is analyzed in detail on three different transparent model fractures; spatiotemporal diagrams are used to characterize the motion of the fibers as well as the time variations of their orientation and their deformation.

Fracture $F 1$ has smooth walls: it is used as a reference and allows for quantitative tests of theoretical and numerical models of the motion of fiber segments and of continuous threads (longer than the fracture). The two other fractures have rough walls: their surfaces have a self-affine geometry like that of real fractured rocks. In model $F 2$, there is a small, non zero, angle between the mean planes of the walls, resulting in a non-zero gradient of the mean aperture transverse to the flow: this wedge-like shape mimics the edge of many natural fractures. In fracture $F 3$, the wall geometry is the same but the mean planes of the surfaces are parallel: this models regions of the fracture away from the edges. Particular attention is devoted the pinning processes observed in the rough models: they will be characterized here quantitatively by the variation of the location and deformation of the fibers with time and the correlation of the location of the pinning sites with the aperture.

\section{Experimental and numerical simulation methods}

\subsection{Fiber characteristics}

The experimental fibers are prepared from commercial polyester thread used for needlework and made of two strands twisted together. The section is not circular and its maximum and minimum transverse sizes are 220 and $340 \mu \mathrm{m}$. The density of the fiber is: $\rho=1.4 \pm$ $0.110^{3} \mathrm{~kg} / \mathrm{m}^{3}$ and its lineic mass: $2.6 \times 10^{-5} \mathrm{~kg} / \mathrm{m}$. Its bending stiffness $J$ (ratio of the applied bending momentum by the curvature) is of the order of $10^{-8} \mathrm{~kg} \cdot \mathrm{m}^{3} / \mathrm{s}^{2}$ (a value similar to that reported in ref. [Habibi et al. 2007] for a comparable material). This value has been estimated by measuring the deflection under its own weight of an horizontal fiber segment attached at one end [Landau and Lifshitz 1986]. It should be noted that the thread has not a perfectly elastic behaviour and may display a plastic deformation.

We used in the experiments fiber segments much shorter than the fracture length $L$ as well as "continuous" fibers longer than $L$ : they are cut out of the same spooled polyester thread sample. A specific procedure is used to cut the thread and keep the strands tightly twisted. The length of the segments is $20 \mathrm{~mm} \leq \ell \leq 150 \mathrm{~mm}$ while the length of the continuous fiber is slightly larger than that of the fracture.

\subsection{Model fractures}

The three models are manufactured with the technique described by [Boschan et al. 2007]:

- $F 1$ has smooth parallel plane walls separated by a fixed distance $\bar{a}=a(x, y)=0.65 \mathrm{~mm}$ (Fig. 1a).

- F2 has tilted rough walls (see Fig. 4).

- F3 is all but similar to $F 2$ excepted that its walls are parallel (see Fig. 6). $F 3$ and $F 1$ have the same mean aperture (i.e. $\bar{a}=0.65 \mathrm{~mm}$ ).

For each model, two parallelepipedic plexiglas blocks are milled into the desired geometry by a computer controlled machine. The two blocks are then clamped together in a position set by the geometry of the sides of the block: these act as spacers leaving a controlled interval between the surfaces for the fluid flow (Fig. 1). 


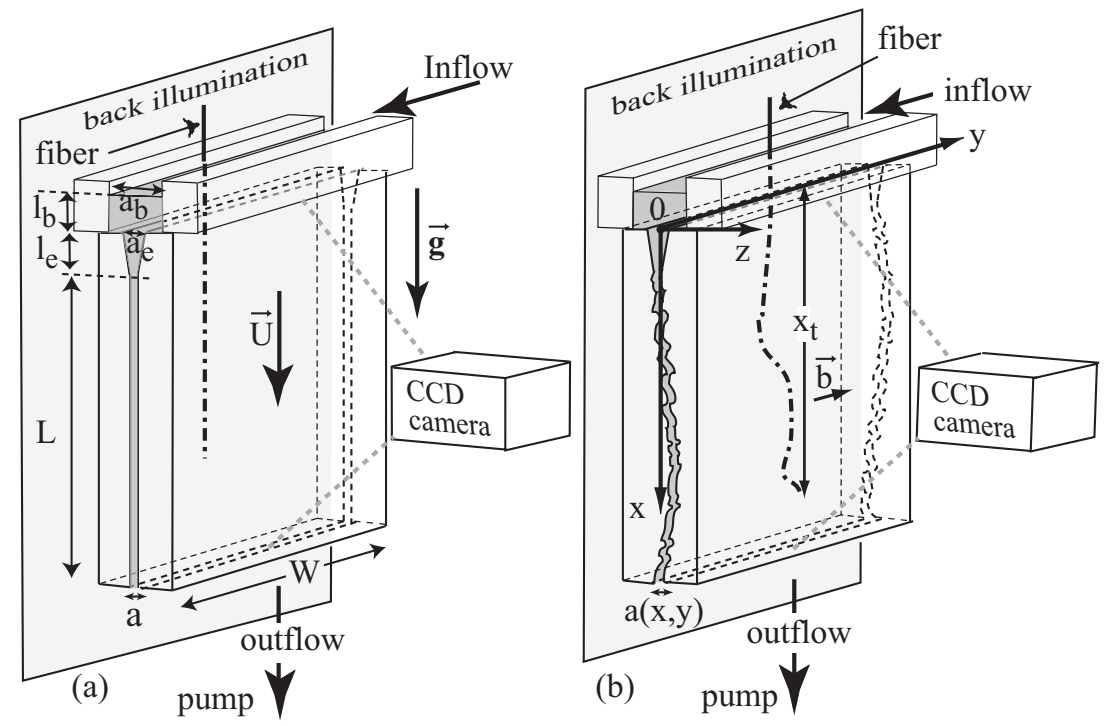

Fig. 1 Schematic view of the experimental models. (a) fracture with flat parallel walls - (b) fracture with complementary self-affine walls with a relative displacement b. For all cells : $W=90 \mathrm{~mm}, a_{b}=20 \mathrm{~mm}$, $a_{e}=5 \mathrm{~mm}, l_{e}=52 \mathrm{~mm}, l_{b} \sim 20 \mathrm{~mm}$ and $L=288 \mathrm{~mm}$. Two injection systems insure that the flow is evenly distributed along $y$ at the top and bottom of the model.

Models $F 2$ and $F 3$ have complementary rough walls with a self affine geometry of exponent $\zeta=0.8$ : this value is similar to that measured on granite fractures [Boffa et al. 1998] and in various other materials [Bouchaud 2003]. The peak to peak amplitude of the roughness, represented by the difference between the extrema of the height of the surface with respect to the mean plane, is equal to $26 \mathrm{~mm}$ : this is also close to values measured on natural rock fractures [Poon et al. 1992]. The surfaces of the two walls are exactly complementary and may be brought into perfect contact: in the models, these surfaces have both a normal displacement with respect to this reference position and a relative shear one: the latter introduces a variability of the local aperture field [Boschan et al. 2007].

The shear component of this displacement introduces local fluctuations in the aperture field: matching features of the roughness of the two walls do not correspond any more to same $(x, y)$ coordinates. Moreover, there appears a structural anisotropy normal to the shear displacement [Gentier et al. 1997, Yeo et al. 1998]. For both fractures $F 2$ and $F 3$, the shear amplitude (parallel to $y$ ) is $u=0.65 \mathrm{~mm}$; the resulting aperture fields have a root mean square amplitude equal to $0.122 \mathrm{~mm}$ and a structural anisotropy oriented along the $x$ direction (see Fig. 6).

In model $F 3$, the mean planes of the walls are parallel and at a fixed distance $\bar{a}=$ $0.65 \mathrm{~mm}$; in model $F 2$, there is between them a small angle mimicking the edge of natural fractures. As a result, the average $\bar{a}(y)=<a(x, y)>_{x}$ of the aperture in the direction $x$ of the flow decreases across model $F 2$ (Fig. 4c) from $1.1 \mathrm{~mm}$ for $y=0$ to $0.8 \mathrm{~mm}$ for $y=W(W$ is the width of the model). This wedge-like geometry of the aperture is reflected by the global color gradient in the direction $y$ for the maps of Fig. 4a-b. 
2.3 Experimental set-up and procedure

The model fractures are held vertically; liquid is sucked uniformly at the bottom side at a constant flow rate and reinjected at the top into an open bath of area $5 \times 90 \mathrm{~mm}$ and depth $l_{b} \sim 20 \mathrm{~mm}$ covering the inlet of the model. This design allows one to introduce the fibers in the bath through its open surface and then into the fracture. In order to ease the injection of the fibers, the upper section has a funnel-like "Y" shape, i.e. the mean aperture of the fracture increases with height in the top $52 \mathrm{~mm}$ of the model (distance $l_{e}$ in Figs. 1a-b). They are progressively injected from above into the fracture with no applied tension.

The model is illuminated from behind by a light panel and a digital camera provides images with $1024 \times 768$ pixels at a rate of 30 frames per second and with an exposure time of $1 / 300 \mathrm{~s}$. The length of the field of view is $150 \mathrm{~mm}$ in the vertical direction parallel to the flow and the top of the aperture maps is at a distance of $110 \mathrm{~mm}$ from the inlet.

On each picture, the location and geometry of the fiber is determined by binary thresholding. For most experiments, the distortions of the fiber remain moderate (except when it gets pinned) and it remains overall aligned with the direction of the flow. Then, at each distance $x$ from the inlet, corresponds only one point of the fiber and, therefore, only one value of $y$. From these data, spatiotemporal diagrams like those displayed in Figs. 2a, 3a and 5a-c are obtained. The vertical scale corresponds to the vertical distance $x$, the horizontal one to time and the color code corresponds to the coordinate $y$ of the fiber at the corresponding value of $x$. Qualitatively, these diagrams provide information on the global motion of the fibers, on their rotation and on their deformation. For instance, diagrams made of segments of same colors and length for all times correspond to a pure translation of the fiber in the direction parallel to the mean flow; in contrast, color variations in the segments imply lateral motions. Quantitatively, the velocity of segments of fibers with a finite length is determined from the variation between two successive images of the vertical distance $x_{m}$ of their center of mass to the inlet of the model. For continuous fibers, one uses instead the variation of the distance $x_{t}$ of the tip of the fiber to the inlet. In both cases, the vertical velocity of the center of mass (respectively tip) of the fiber is referred to as $V_{f}$.

\subsection{Characteristics of the fluids}

Two fluids are used in these experiments. The first one is high purity water (Millipore - MilliQ grade) with a density $\rho=10^{3} \mathrm{~kg} / \mathrm{m}^{3}$ and a dynamic viscosity $\mu_{\infty} \simeq 1 \mathrm{mPa}$.s. The second fluid is a solution of high molecular weight Scleroglucan (Sanofi Bioindustries) in water at a concentration $C_{p}=1000 \mathrm{ppm}$. This fluid has shear thinning characteristics similar to those of fluids used in oil recovery; the variation of its effective $\mu$ with $\dot{\gamma}$ ([D'Angelo et al. 2007], Fig. 2) follows well the Carreau function:

$$
\mu=\frac{1}{\left(1+\left(\dot{\gamma} / \dot{\gamma}_{0}\right)^{2}\right)^{\frac{1-n}{2}}}\left(\mu_{0}-\mu_{\infty}\right)+\mu_{\infty} .
$$

with $n=0.26, \mu_{0}=4500 \pm 340 \mathrm{mPa} . \mathrm{s}$ and $\dot{\gamma}_{0}=0.026 \pm 0.004 \mathrm{~s}^{-1}$. Here, the mean flow velocity ranges from $U=50$ to $400 \mathrm{~mm} / \mathrm{s}$ : so, the typical shear rate $\dot{\gamma}=6 U / \bar{a}$ is of the order of $1000 \mathrm{~s}^{-1}$, i.e. much above $\dot{\gamma}_{0}$. The Carreau approximation predicts an effective viscosity $\mu=3 \mathrm{mPa}$.s (i.e. 3 times that of water) for $U=160 \mathrm{~mm} / \mathrm{s}$. For water, the corresponding Reynolds number defined as $R e=U \bar{a} \rho / \mu$ varies from 40 and 320: the influence of the inertial terms will very low in the smooth models due to the quasiparallel flow geometry 
but may more substantial in the rough case, particularly when the relative variations of the aperture (inducing velocity gradeints) are large [Zimmerman et al. 2004]. An alternative value of the Reynolds number, more relevant to the estimation of the forces on the fibers, is obtained by using the diameter of the fiber and its relative velocity with respect to the fluid; it is however still higher than 1 .

\section{Fiber transport in a fracture with smooth plane walls}

3.1 Transport of fiber segments
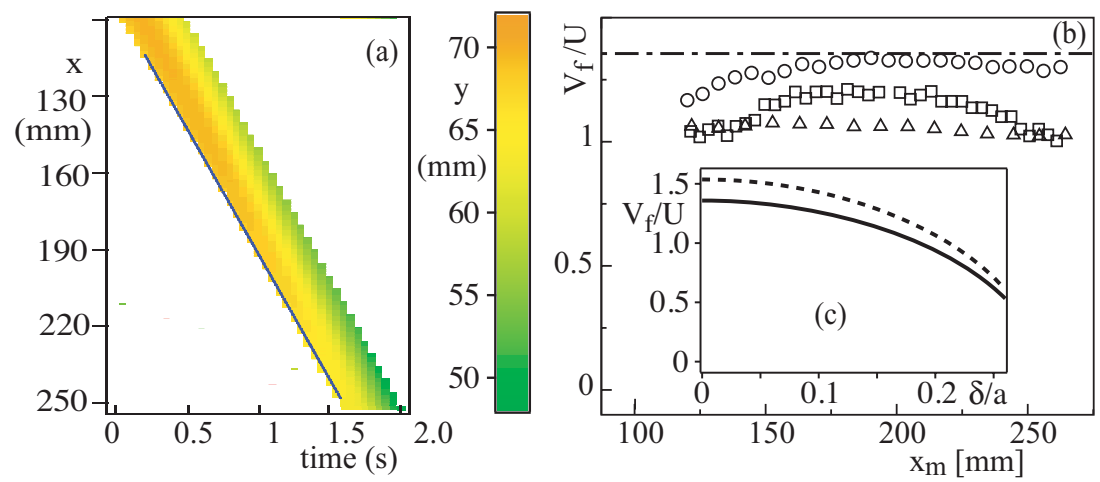

Fig. 2 a) Spatiotemporal diagram of the motion of a fiber segment of length $\ell=20 \mathrm{~mm}$ as a function of time in model $F 1$. Vertical scale: vertical distance $x$ from the top inlet of the mode; color shades: transverse coordinate $y(x)$ of the points of the fiber (see scale at right). b) Variation of the velocity $V_{f}$ of the center of mass of fiber segments of length $\ell=20 \mathrm{~mm}$ as a function of $x_{m}:(\square) U=100 \mathrm{~mm} / \mathrm{s}$, (०) $U=150 \mathrm{~mm} / \mathrm{s}$, $(\triangle) U=290 \mathrm{~mm} / \mathrm{s}$. Dash-dotted line: maximum observed value of $V_{f} / U=1.35$. Inset: normalized velocity obtained from numerical simulations for a cylinder moving freely in a parallel flow; dotted (resp. continous) lines include (resp. neglect) the influence of buoyancy.

In order to separate the influence of the generic fracture geometry and of the roughness of the walls, reference experiments were carried out in model $F 1$ with flat smooth walls. A first set of experiments used fiber segments of length $\ell$ ranging from 20 to $150 \mathrm{~mm}$ and mean fluid velocities: $50 \leq U \leq 400 \mathrm{~mm} / \mathrm{s}$. For each set of values of $\ell$ and $U$, more than 20 transport experiments were realized. In the following, we discuss the transport of fibers with their mean axis within $\pm 10^{\circ}$ to the direction of the flow.

In all cases, no deformation of the fibers is visible during the motion. The spatiotemporal diagram of Figure 2a indicates first that the velocity component along $x$ of the ends of the fiber remains constant along its trajectory (straight boundaries of the colored region) and that there is only a small drift along $y$ (slow shift of the colors). This is confirmed quantitatively by Figure $2 \mathrm{~b}$ in which the normalized longitudinal velocity $V_{f} / U$ of the center of mass of the fiber is plotted as a function of its distance $x_{m}$ to the inlet for 3 different values of $U$ (open symbols) in a range of 1 to $3 . V_{f} / U$ is constant with $x$ within $\pm 10 \%$; its variation with $U(\leq 25 \%)$ is also too small to suggest any definite dependence in view of the intrinsic dispersion due to the injection process and to the variability of the shape of the fiber. More generally, in other experiments performed at different velocities and for fibers of different 
lengths, $V_{f} / U$ always ranges between 1 and 1.35 (the velocity $V_{f}$ is therefore always lower than the maximum value $1.5 V_{f}$ of the velocity of the fluid in the gap).

We compared these experimental observations to numerical simulations in which the fiber is modeled as a straight rigid cylinder of similar diameter. Except for the numerical work of [Frei et al 2000], all studies of moving cylinders in confined geometry were restricted to the case of cylindrical objects normal to the flow [Bouard 1997, Zovatto et al 2001, Richou et al 2005]. Finite element simulations were thus undertaken to determine the velocity $V_{f}$ of a fiber parallel to the flow; particular attention was given to the dependence of $V_{f}$ on the distance of the axis of the cylinder from the mid-plane of the fracture. Assuming that the flow is laminar and that the influence of the ends of the cylinder is negligible, the only non zero component of the fluid velocity $\boldsymbol{U}$ is parallel to $\boldsymbol{e}_{\boldsymbol{x}}$ and is given by $\boldsymbol{U}=U(y, z) \boldsymbol{e}_{\boldsymbol{x}}$. In this case, the Navier-Stokes equation reduces to a $2 D$ Laplace equation. The latter is solved by means of the finite element program FreeFem++ with zero velocity boundary conditions on the lateral side plates at $y= \pm W / 2$ and on the front plates at $z= \pm a / 2$. The forces acting on the cylinder have then been computed both when it is translating at a constant velocity along the fracture in a stationary fluid and when it is held fixed in a Poiseuille flow of mean velocity $U$. The two solutions are then superimposed to obtain the velocity $V_{f}$ of the cylinder in a Poiseuille flow and with no applied force.

The solid line in the inset of Fig. $2 b$ displays the variation of the normalized velocity $V_{f} / U$ with the normalized distance $\delta / a$ between the axis of the cylinder and the mid-plane of the fracture. As $\delta / a$ increases from 0 (cylinder in the mid-plane), the normalized velocity continuously decreases from 1.35 to a value close to 0.8 at the closest distance from the fracture walls (i.e. for $\delta / a \sim 0.23$ ). The density difference between the fiber and the fluid may also influence the velocity of the fiber: the influence of this buoyancy effect on the velocity $V_{f} / U$ is obtained using the same numerical procedure as above. The dashed line in Fig. $2 \mathrm{~b}$ corresponds to the values obtained in this case which are, at most, $10 \%$ higher.

The comparison of these numerical simulations to the experimental data (Fig.2b) shows that the axis of the cylinder is likely located in the central half of the fracture aperture. The variations of the velocity $V_{f}$ with the distance $x_{m}$ and/or the velocity $U$ observed experimentally may be due to displacements of the fiber in the fracture aperture or to deviations of its shape from a straight line.

\subsection{Transport of continuous fibers}

In the above experiments, the drag forces could be assumed to remain constant along the relatively short fiber segments which were studied. We consider now the case of fibers with a length larger than that of the model, lying initially on its top side, and dragged by the flow into the fracture once its tip has been inserted inside it. These fibers are referred to in the following as "continuous". In this case, the flow velocity and, therefore, the drag force are lower in the upper part of the fracture (i.e. in the upper bath and in the " $Y$ " shape domain displayed in Fig.1).

Figure 3a displays the spatiotemporal diagram of such an experiment. At a given time $t$, the color is constant along $x$ in the colored zone, indicating that the fiber remains straight and vertical. The color varies slightly with time which reflects a small global transverse displacement. The boundary of the colored zone marks the variation of the coordinate $x_{t}$ of the tip with the time $t$ : its slope (proportional to the velocity $V_{f}$ of the tip) increases with $x_{t}$. This trend is confirmed quantitatively by Fig. $3 \mathrm{~b}$ (symbols) in which the normalized velocity $V_{f} / U$ is plotted as a function of $x_{t}$. In a simple model, the fiber is assumed to: a) 

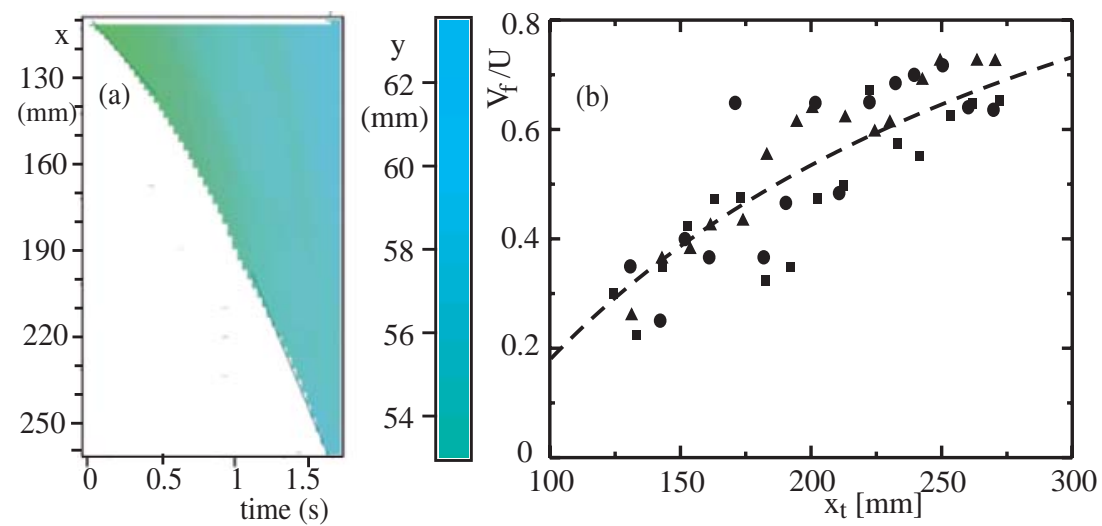

Fig. 3 a) Spatiotemporal diagram of the motion of a continuous fiber as a function of time in model $F 1$ for $U=120 \mathrm{~mm} / \mathrm{s}$. Vertical scale $=$ vertical distance $x$ from the top inlet of the model. Color shades: transverse coordinate $y(x)$ (see scale at right) - (b) Variation of the normalized velocity $V_{f} / U$ of the tip of the continuous fiber as a function of the distance $x_{t}:(\boldsymbol{\square}) U=100 \mathrm{~mm} / \mathrm{s},(\bullet) U=150 \mathrm{~mm} / \mathrm{s},(\boldsymbol{\Delta}) U=290 \mathrm{~mm} / \mathrm{s}$. Dashed line shows Eq. 4 with $\Delta=860 \mathrm{~mm}$.

remain straight and vertical so that the velocity of all its points is equal to $V_{f}$ and: b) be located in the center of the gap (this is indeed approximately the case for short fibers as shown in the previous section). Friction of the fiber with the walls is neglected.

Under the assumption that the flow is everywhere laminar and parallel to $\boldsymbol{e}_{\boldsymbol{x}}$ the equation of motion is linear and the local vertical force $f_{v}$ per unit length of the fiber in the cases of a stationary fiber $\left(V_{f}=0, U \neq 0\right)$ and of a stationary fluid $\left(V_{f} \neq 0, U=0\right)$ would respectively be proportional to $U$ and $V_{f}$ [Guyon et al. 2001]. By superimposing the two flows, one obtains in the general case:

$$
f_{v}=\mu\left(c(x) U-c_{f}(x) V_{f}\right)
$$

in which $c$ and $c_{f}$ are geometrical coefficients depending on the local width of the flow channel at the distance $x$ and $U$ is the velocity in the constant aperture domain. The numerical procedure described in the previous section has been used to determine the coefficients $c$ and $c_{f}$ at different distances $x$ for a fiber located mid-way between the walls. The local values of $c$ and $c_{f}$ found in this way for a $0.28 \mathrm{~mm}$ fiber are respectively: $c_{f}=2.01$ and $c=0.41$ in the upper bath and $c_{f}=5.81$ and $c=7.90$ in the constant aperture domain $(\bar{a}=0.65 \mathrm{~mm})$. The average values in the Y-shaped funnel are $c_{f}=2.79$ and $c=1.45$. The variation of local flow velocity with the local aperture has been taken into account in the determination of $c(x)$. Summing the contribution of the three zones leads to the total vertical force:

$$
F_{v}=\mu\left(C\left(x_{t}\right) U-C_{f}\left(x_{t}\right) V_{f}\right)
$$

$x_{t}$ is the coordinate of the tip of the fiber and $C$ and $C_{f}$ are given by: $C\left(x_{t}\right)=7.81 x_{t}-$ $6.36 l_{e}+0.41 l_{b}, C_{f}\left(x_{t}\right)=5.81 x_{t}-3.02 l_{e}+2.01 l_{b}$. An additional drag force $F$ results from the motion of the residual length of fiber which is stored in the upper bath before being fed into the fracture at later times. Neglecting the influence of the flow in the upper bath on this part of the fiber, we assume that this drag force is proportional to the velocity $V_{f}$ and to the effective length $\Delta-x_{t}-l_{b}$ of this part of the fiber ( $\Delta$ is an effective length). For a fiber moving freely at a nearly constant velocity, the sum of all forces must be zero with: 
$F+F_{v}=\mu\left(C\left(x_{t}\right) U-C_{f}\left(x_{t}\right) V_{f}-\left(\Delta-x_{t}-l_{b}\right) V_{f}\right)=0$. The ratio $V_{f} / U$ is then:

$$
\frac{V_{f}}{U}=\frac{C\left(x_{t}\right)}{C_{f}\left(x_{t}\right)+\Delta-x_{t}-l_{b}}
$$

In the previous equation, the only free parameter is $\Delta$. The latter was determined by fitting the experimental data points of Fig. $3 \mathrm{~b}$ (dotted line). The adjustment of the data is good for $\Delta=860 \mathrm{~mm}$, i.e. more than the length of the fiber $(\simeq 600 \mathrm{~mm})$. This large value may arise from extra friction due to loops and to the contact between the fiber and the walls in the upper bath.

The inertia of the fiber may also need to be taken into account in the acceleration phase. The corresponding term of the equation of motion of the fiber is $m a$ ( $m$ and $a$ are its mass and acceleration); it may be estimated from $m a \approx \ell \lambda V_{f}^{2} / x_{t}$ in which $\lambda=2.6 \times 10^{-5} \mathrm{~kg} \cdot \mathrm{m}^{-1}$ and $\ell \sim 0.6 \mathrm{~m} ; V_{f} \sim 0.15 \mathrm{~m} . \mathrm{s}^{-1}$ and $x_{t} \sim 0.2 \mathrm{~m}$ are the typical length and velocity of the fiber and the distance over which it accelerates. This leads to the value $m a \approx 18 \times 10^{-7} \mathrm{~N}$ which may be compared to the typical hydrodynamic force $F=C_{f} U L \mu$. Using the value $C_{f} \sim 8$ obtained from numerical simulations gives $F \approx 2 \times 10^{-4} \mathrm{~N}$ : this is several orders of magnitude larger than the inertial term which validates the quasi static assumption used up to now.

\section{Fiber transport in model fractures with rough walls}

The above experiments in smooth fracture models demonstrate a negligible interaction of the fibers with the walls; in rough fractures, instead, both the random variations of the aperture and the large out of plane deformations of the wall surfaces induce large friction forces. These forces influence the motion of the fibers and may also deform them: we shall show that these effects depend both on the fluid velocity and on the rheological properties of the fluid.

\subsection{Fiber transport in the wedge-shaped fracture (model $F 2$ )}

A first set of experiments has used water as the flowing fluid: in this case, fiber transport was only possible in regions of aperture $\gtrsim 1 \mathrm{~mm}$, (i.e. on the left side of the maps of Fig. 4a-b). In lower aperture regions, most fibers got pinned after a short distance. Moreover, in a few experiments performed at high flow rates $(U \geq 250 \mathrm{~mm} / \mathrm{s})$, a loop built up downflow of the pinning site (Fig. 4b). Then, the bottom of the loop slid sideways into a higher aperture region and kept moving downwards toward the end of the model.

Even in the high aperture paths, the motion of the fiber is not as continuous as in the smooth fracture as can be seen by comparing the spatiotemporal diagrams of Figs. $3 \mathrm{a}$ and $5 \mathrm{a}$ (the latter corresponds to the same region as in Fig. 4a). This time, the motion of the tip is a sequence of "stop" and "go" phases marked respectively by horizontal and oblique sections of the boundary of the colored zone in Fig. 5a.

The influence of the flow velocity on this "stop" and "go" character of the motion is analyzed in Figure 5b: it compares the variations with distance of the normalized velocity $V_{f} / U$ of the fiber tip for two different values of $U$. At the lower flow velocity $U=160 \mathrm{~mm} / \mathrm{s}$, the tip velocity $V_{f}$ becomes zero at each pinning point and increases sharply after the release of the fiber to a value higher than the time average (horizontal dashed line). For $U=210 \mathrm{~mm} / \mathrm{s}$, 


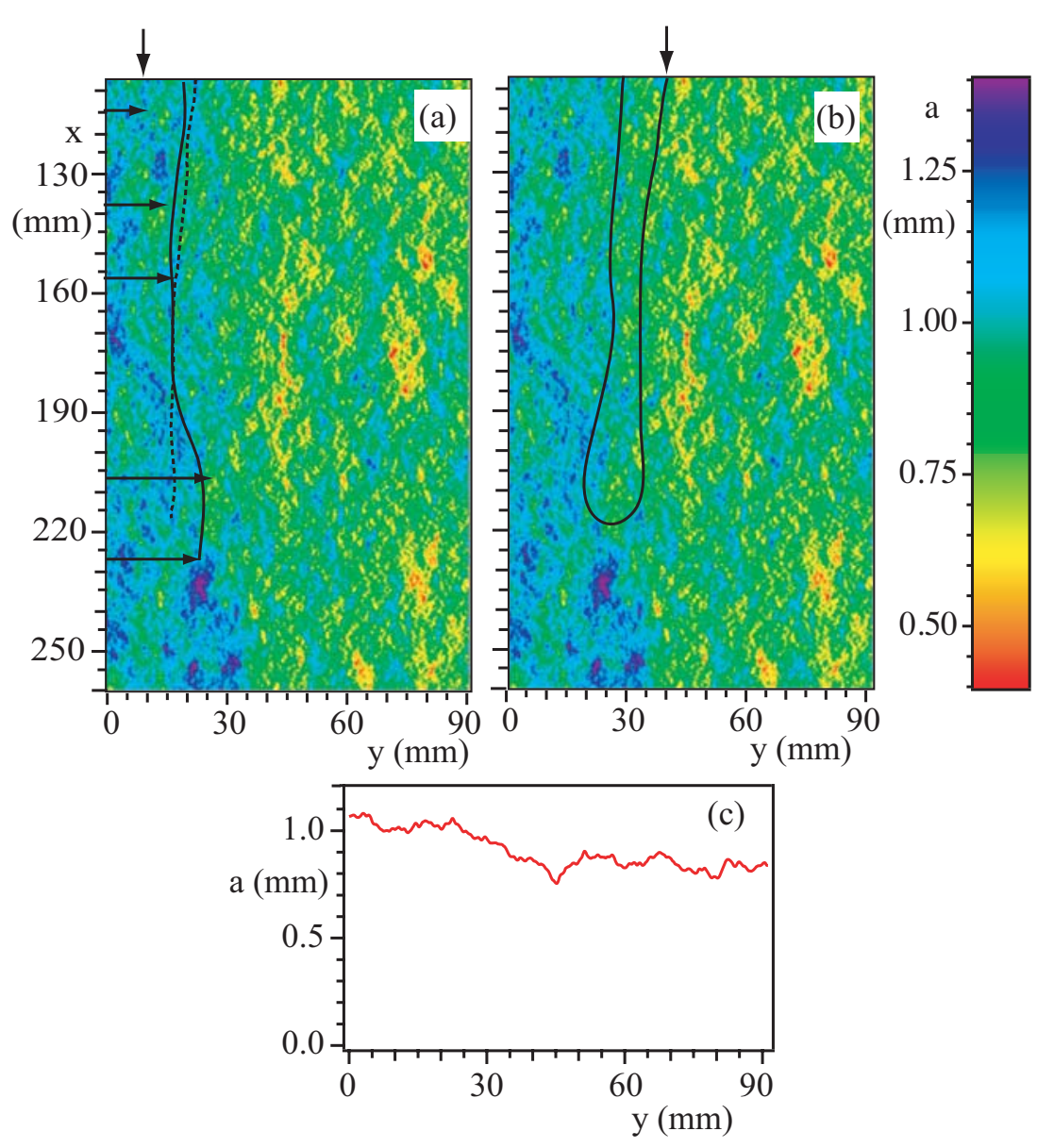

Fig. 4 (a-b)Snapshots of experiments in which the continuous fiber is injected in a water or polymer flow in model $F 2$ at different transverse distances (vertical arrows). The images of the fiber are superimposed onto a color coded map of the aperture field (see scale at right). Field of view: $90 \times 150 \mathrm{~mm}$. (see Fig. 1). (a) $U=160 \mathrm{~mm} / \mathrm{s}$. Continuous line: water flow (horizontal arrows corresponds to the 5 locations where the fiber was temporarily pinned during its motion); dotted line: polymer flow. (b) $U=250 \mathrm{~mm} / \mathrm{s}$ : water flow. (c) variation of the average $\bar{a}(y)$ of the aperture along the distance $x$ parallel to the flow as a function of the transverse distance $y$.

the variations with time of the fiber velocity with respect to the mean value are of smaller amplitude $(-100 \%,+50 \%)$ instead of $(-100 \%,+150 \%)$. Also, the velocity $V_{f}$ becomes zero only once and decreases by less than $40 \%$ on other pinning sites: these are located at the same distances $x$ as for $U=160 \mathrm{~mm} / \mathrm{s}$. These results demonstrate that increasing the velocity reduces pinning effects but that they remain strong. The inertia of the fibers might act in an opposite way by pushing them away from the streamlines and toward the walls. This would increase the number and the influence of the contacts of the fiber with the walls: this is not observed which implies that the inertia of the fibers does not play a dominant part.

More quantitatively, the normalized time averages of the fiber velocity $\overline{V_{f}} / U$ are equal to 0.2 and 0.28 (respectively for $U=160$ and $210 \mathrm{~mm} / \mathrm{s}$ ). Even if the influence of the low velocities near the pinning sites is subtracted from these values, $\overline{V_{f}} / U$ is still only of the 

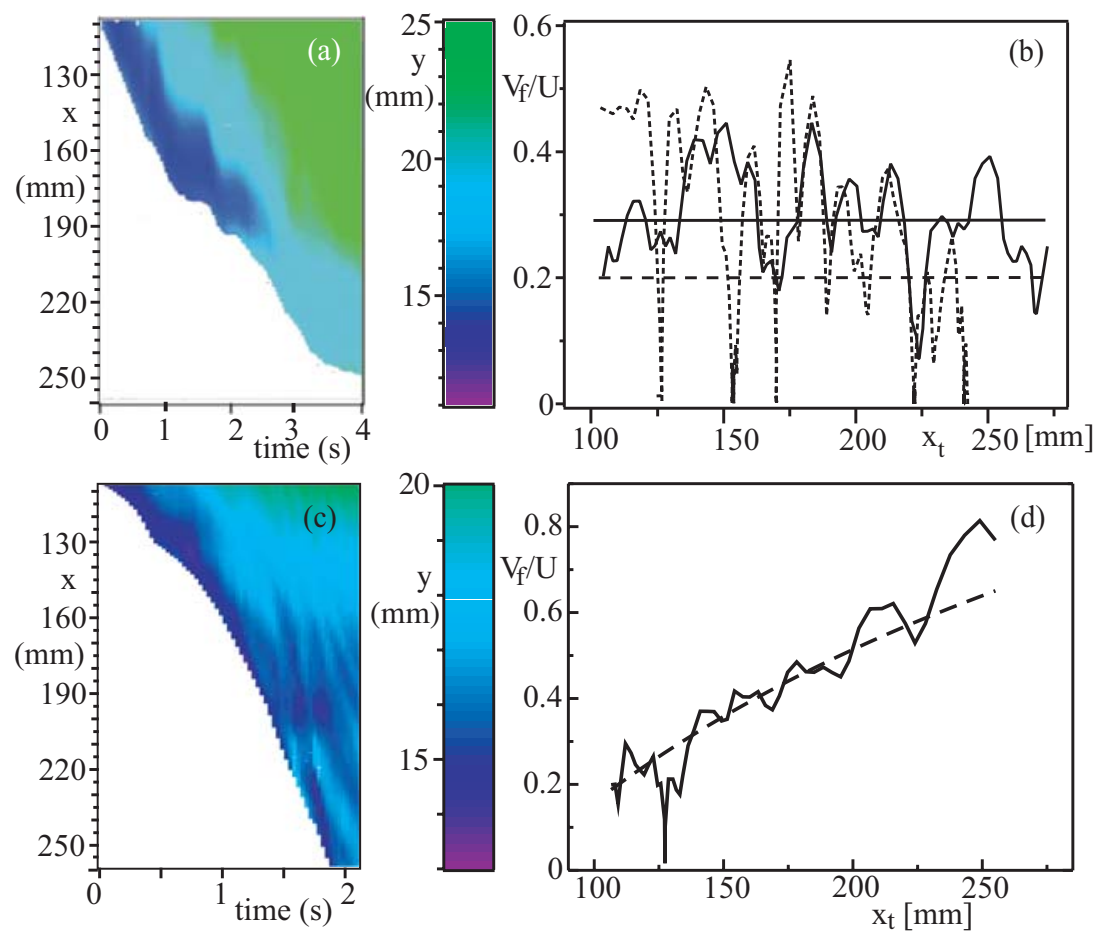

Fig. 5 Compared motion of continuous fibers as a function of time in model $F 2$ for flows of water (a,b) and of a polymer solution (c,d). (a,c) Spatiotemporal diagrams of the motion in $x, t$ coordinates at $U=160 \mathrm{~mm} / \mathrm{s}$. Color shades: : transverse coordinate $y(x)$ (see scale at right of the corresponding diagram). (b,d) Variation of the normalized fiber velocity $V_{f} / U$ as a function of the distance $x$ for a same initial transverse location of the fibers. (b) dotted (resp. continuous) lines: $U=160$ (resp. 210 ) $\mathrm{mm} / \mathrm{s}$. (d) continuous line: $U=220 \mathrm{~mm} / \mathrm{s}$. Dashed line: fit with Eq. $4\left(\Delta=790 \mathrm{~mm} ; c_{f}=0.70, c=2.00\right.$ in the upper bath; $c_{f}=5.71, c=3.87$ in the region of aperture $\bar{a}=1.1 \mathrm{~mm}$ constant with $x ; c_{f}=1.80, c=2.57$ in the Y-shaped funnel (averaged values)).

order of 0.3 in both cases: this is lower by a factor of 3 than in model $F 1$ (see Fig. 3) which confirms the strong interaction of the fibers with the rough walls. This conclusion is supported by the lack of systematic dependence of $V_{f}$ on the distance $x$ : this latter effect suggests that the influence of the inlet is screened by the friction at the walls.

The above experiments have been repeated at similar flow rates, but using the polymer solution described in Sec. 2.4 which represents well many fluids used in practical industrial applications. First, the corresponding spatiotemporal diagram (Fig. 5c) does not display the stop and go features observed in Fig. 5a; then, the velocity $V_{f}$ increases continuously with the distance $x$ (Fig. 5d). Overall, this variation is more similar to that observed for water flows in the smooth model $F 1$ (Fig. 3b) than in model $F 2$ (Fig. 5b); compared to this case, the variations of the color shades indicate however additional deformations and sideways motions of the fiber. More quantitatively, at long enough distances, the ratio $V_{f} / U$ for model $F 2$ is much larger for the polymer solution than for water $\left(V_{f} / U=0.8\right.$ instead of 0.3 for $x_{t}=250 \mathrm{~mm}$ ). These experimental data may be fitted by the predictions of Eq.4 (dashed line in Fig. 5d) applied previously to the smooth fracture; the interactions with the walls are still neglected and new coefficients $c$ and $c_{f}$ are used to take into account the different aperture distribution. The optimal value of $\Delta$ is now $790 \mathrm{~mm}$ which is similar to that found 
for the smooth fracture. Finally, while the velocity of the fiber still drops when it reaches the pinning sites observed in the experiments with water, the amplitude of the variation is far lower.

The above results indicate that the relative influence of the friction with the walls on the dynamics of the fiber is significantly reduced when the polymer solutions are used. This may reflect an enhancement of the hydrodynamic drag forces and/or a reduction of the friction forces. As a result, the tension forces are not fully balanced by friction and propagate along the fiber. These forces influence the shape of the fibers which is significantly straighter than for water: this is shown by Fig.4a in which images obtained for flows of the two different solutions are overlaid.

\subsection{Fiber transport in a fracture with rough parallel walls (fracture $F 3$ )}

In the case of model $F 2$, the "wedge like" geometry allows one to test the influence of the mean aperture $\bar{a}$ by injecting the fiber at several transverse distances $y$ corresponding to different $\bar{a}$ values. However, this geometry may have unwanted effects like the sideways motions displayed in Fig. 4. Such effects are not present in model $F 3$ which has a constant mean aperture $\bar{a}=0.65 \mathrm{~mm}$. In the case of a water flow, transport of the fiber across model $F 3$ was not possible since it always got pinned irreversibly along its path: this was to be expected since the mean aperture $\bar{a}$ is lower than the threshold value estimated in the previous section for model $F 2$. All fiber transport experiments in model $F 3$ were therefore always performed with the polymer solution discussed above.

In Figure 6a, snapshots of four fibers injected at different transverse distances $y$ in model $F 3$ are overlaid onto the aperture map. Three of them are weakly deformed and kept moving afterwards down to the lower end of the model. Their location is close to (but does not coincide exactly with) the zone of highest aperture. The fourth fiber (at the left on the image) got pinned as it moved out of a high aperture region into a less open one; the shape of this fiber is also more strongly distorted than that of the others, possibly due to large velocity gradients in the region where the fiber moves.

Overall, these experiments on model $F 3$ demonstrate the feasibility of the transport of the fiber in a model with parallel mean walls, even in the case of a small mean aperture $(2.5$ times the mean fiber diameter). This transport is however only possible for this value of the aperture when the polymer solution is used as the flowing fluid. Moreover, the observations of the shape of the fibers, of their paths and of the pinning sites suggest that, while the local aperture is a key factor of the possible transport of fibers inside the fractures, it is not the only one. The spatial gradients of the aperture (often related to the velocity gradients in the plane of the fracture), the slopes of the individual surfaces and, possibly, the inertia of the fiber may also play an important part.

\section{Pinning-depinning dynamics}

The experiments using models $F 2$ and $F 3$ have demonstrated the important influence of pinning on the transport of the fibers in rough fractures. In the present section, we investigate in more detail the relation between the pinning sites and the local geometry of the fractures as well as the dynamics of the pinning and depinning (if any) processes.

In the case of a fiber transported by a water flow in fracture $F 2$, the pinning sites are shown by horizontal arrows in Fig. 4a. It must be noted that pinning is always initiated at the 

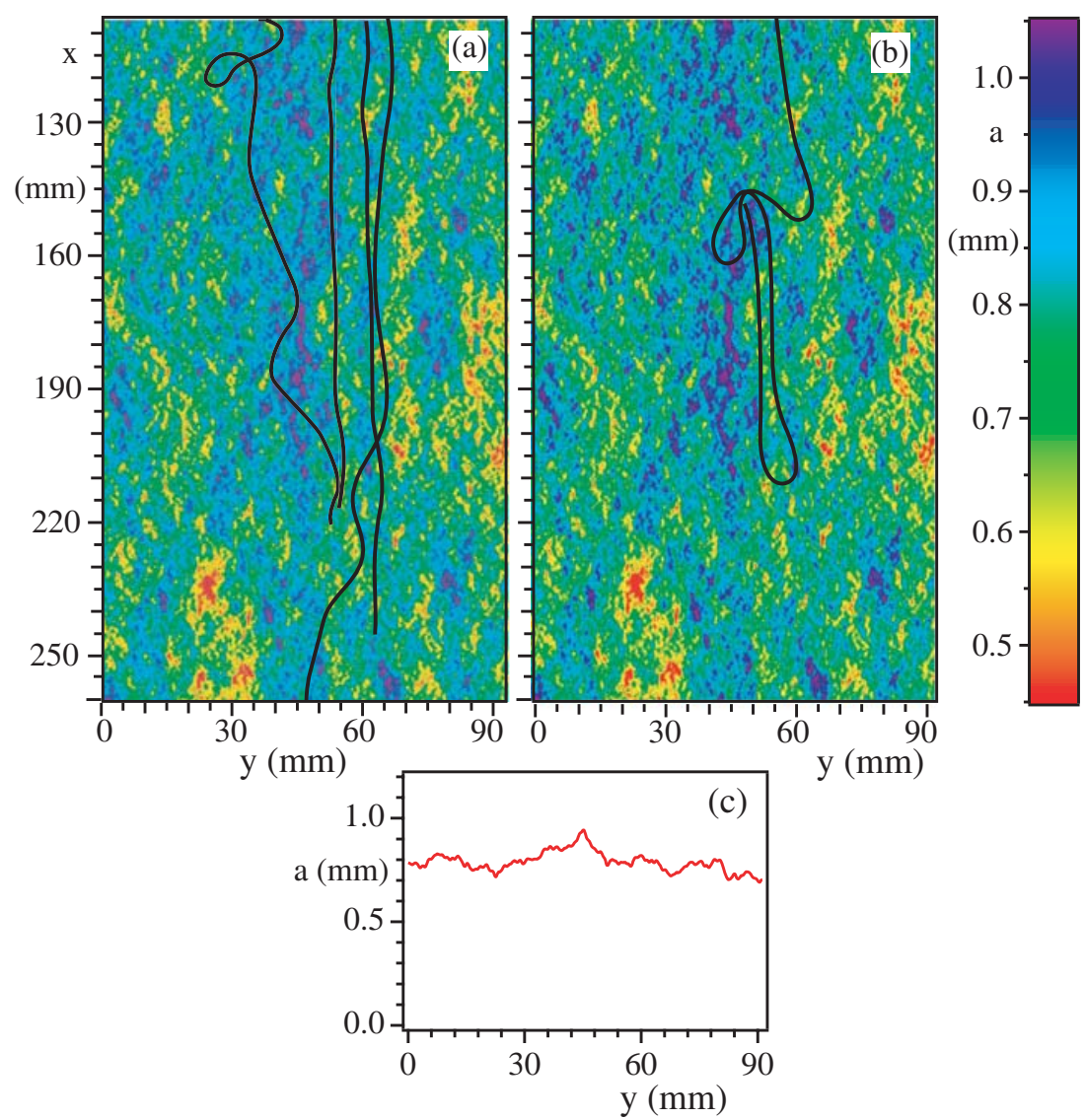

Fig. 6 (a) Overlaid snapshots of 4 fibers injected at different transverse distances into a polymer flow inside fracture $F 3$ and of a map of the aperture field (mean fluid velocity $U=215 \mathrm{~mm} / \mathrm{s}$ ). (b) Snapshot of a fiber coiled up after getting pinned inside the fracture $(U=242 \mathrm{~mm} / \mathrm{s})$. A time sequence of the coiling process is displayed in Fig. 9. The color code is shown in the bar at the top right. Distances in the vertical $x$ direction are counted from the top of the model (see Fig. 1). (c): variations of the aperture averaged along the distance $x$ parallel to the flow as a function of the transverse distance $y$.

tip at the fibers. Several (but not all) pinning sites are located in regions where the aperture is below $1 \mathrm{~mm}$ (yellow-green shades in Fig. 4a). This is still twice the diameter of the fiber so that pinning cannot result solely from a geometrical blockage in these constrictions; moreover, the fiber was observed to cross without stopping other regions of the fracture of similar aperture. As suggested in the previous section, the local aperture is therefore not the only relevant parameter: the analysis of the geometry of the wall surfaces around the pinning points suggests that the blockage of the fibers may result from a large local curvature (out of plane roughness) of the surfaces. In this case, the hydrodynamic forces may be too small to induce enough bending of the fibers so that they follow the surface without touching it.

Further information on the pinning-depinning process is obtained from the deformation of the fiber. Color variations with time and distance in Fig. 5b indicate that, in contrast to the case of a smooth fracture (Fig. 3a), the fiber does not remain straight but buckles with meanders of shape and location varying with time. These deformations are visualized 

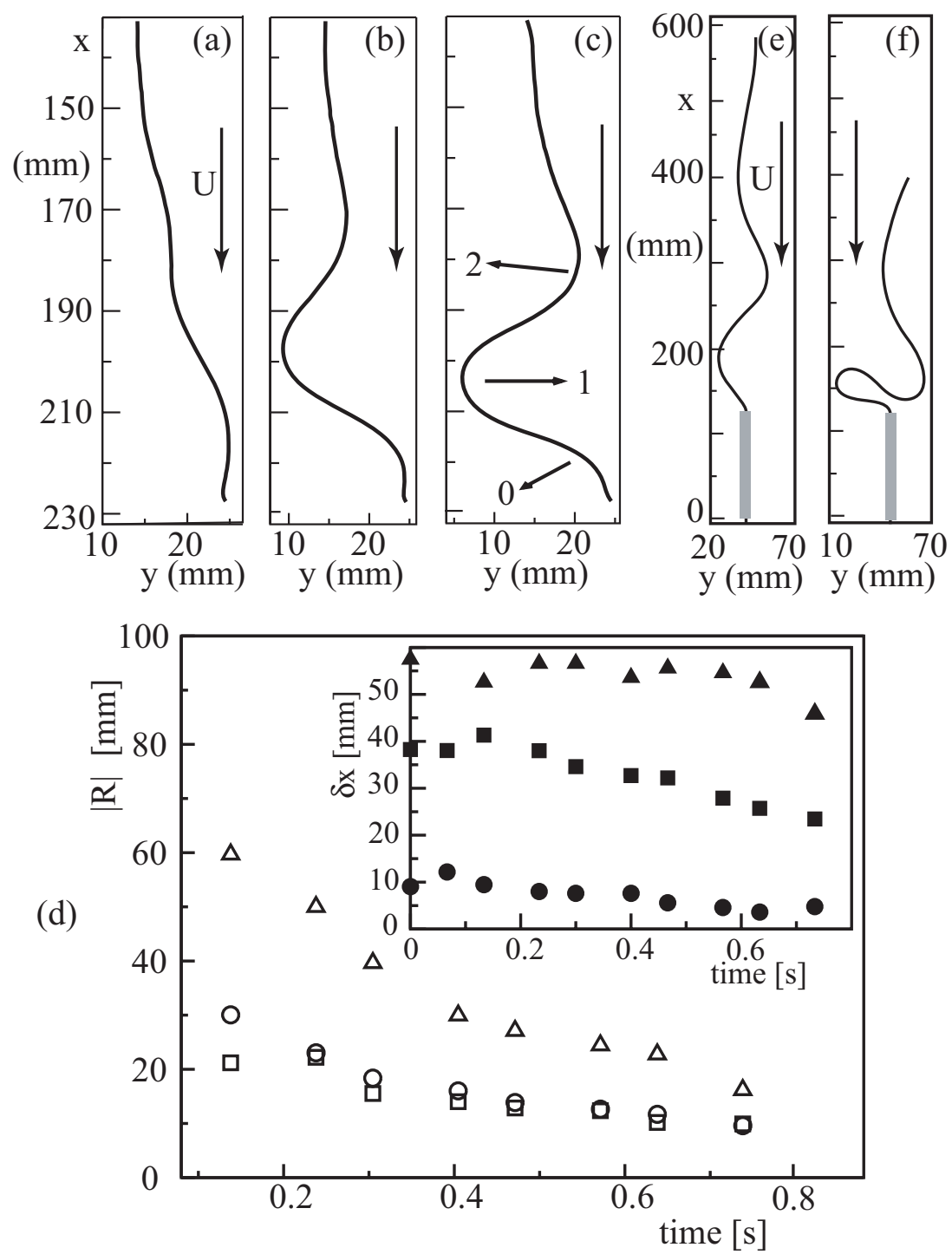

Fig. 7 (a, b, c): Fiber geometry at different times during the fifth pinning event in Fig. 4a (lowest horizontal arrow). The leftmost (respectively rightmost) picture displays the shape of the fiber immediately after pinning (respectively before depinning). Time lapse between pictures: $0.166 \mathrm{~s}(\mathrm{a}-\mathrm{b})$ and $0.333 \mathrm{~s}(\mathrm{~b}-\mathrm{c})$; total duration of trapping event: $1 \mathrm{~s}$. Arrows: location of points of maximum curvature referred to as 0,1 and 2. (d) Curvature of the fiber at these 3 points as a function of time. Inset: vertical distances $\delta x$ between these 3 points and the fiber tip as a function of time. Symbols for point $0:(\circ, \bullet)$; for point $1:(\square, \boldsymbol{\square})$ and for point $2:(\triangle, \mathbf{\Lambda})$.(e,f) View at two different times of an experiment in a cell with smooth plane parallel walls. The fiber is attached to the end of a $1 \mathrm{~mm}$ diameter rod (vertical gray rectangle). Flow is upwards but the pictures have been flipped upside down to make comparisons with Figs. 7a-c easier.

directly in Figures 7a-d. During the pinning event, the tip of the fiber remains motionless while three bumps appear behind it: their amplitude increases with time while they propagate 
toward the tip. The deformation of the rear part of the fiber is much weaker but, in some cases, it slips sideways.

An important feature is the fact that the deformation does not extend along the whole fiber but is, instead, localized in a few bumps behind the pinning site: this observation has analogies with the build up of circular coils after a vertical flexible rope moving downwards has reached an horizontal solid plane [Habibi et al. 2007]. We investigated whether this effect was due to the roughness of the walls by realizing an additional model experiment. A fiber hangs below a cylindrical vertical rod inside a cell with flat walls similar to Fracture 1 but with a larger aperture $a=5 \mathrm{~mm}$. When the fluid is at rest, the fiber is flat and vertical; then, an upwards flow is induced and the end of the rod acts as a pinning site. Figures 7e-f show that the geometry of the deformation of the fiber is very similar to that observed in the rough fracture. Meanders appear close to the pinning point while the rest of the fiber has a lower curvature: the similar results obtained in the two cases imply that the wall roughness has not a major influence on the geometry of the distortions of the fiber due to pinning.

In order to understand better the mechanism of these distortions, the experimental findings will now be compared to the model case in which the fiber is considered as an ideal homogeneous elastic rod. The rod is initially straight and vertical with a fixed lower end and a free upper one. The force per unit length $f_{v}$ on the rod is assumed to be constant along its length and to result solely from the hydrodynamic forces; since the rod is fixed, $V_{f}=0$ so that, from Eq. (2): $f_{v}=\mu c(x) U$. Assuming that the initial buckling deformation is localized to a segment of length $L_{m}$ right above the fixed end point, the minimum value of $L_{m}$ for observing buckling induced by the hydrodynamic forces along it is, from [Timoshenko and Gere 1962]:

$$
L_{m} \simeq 2\left(\frac{J}{f_{v}}\right)^{1 / 3},
$$

in which $J$ is the bending stiffness of the fiber: $J \approx 10^{-8} \mathrm{~kg} \cdot \mathrm{m}^{3} \cdot \mathrm{s}^{-2}$ (see Sec. 2.1). The value of $L_{m}$ has been computed for the experiment of Fig. 7 with $c=3.87$ determined as in Sec. 3.2 but for a local aperture $a=1.1 \mathrm{~mm}$. One obtains $L_{m} \sim 50 \mathrm{~mm}$. This is close to the curvature observed experimentally at the onset of buckling (see squares in Fig. 7d). Actually, the buckling process is also modified by the complex structure of the flow field and by the influence of hydrodynamic forces perpendicular to the fiber [Richou et al 2005]. Another important issue is the deformations of the fiber in the direction perpendicular to the mean plane of the fracture: as a result new contact points may appear along the fibers and result in additional local friction forces. These forces will, in turn, influence the transmission of forces along the fiber and, therefore, the depinning process.

The dynamics of the deformation is characterized quantitatively by the variations with time of the radius of curvature $|R|$ at the points of maximum curvature and of their distance $\delta x$ to the tip of the fiber (Fig. 7d): the radii $|R|$ decrease strongly with time while the velocities $d \delta x / d t$ of all three corresponding points are similar and of the order of $10 \mathrm{~mm} / \mathrm{s}$ (i.e. much lower than the fluid velocity $\simeq 160 \mathrm{~mm} / \mathrm{s}$ ). The deformation is stronger for the two first bumps with a radius of curvature of the order of $9.5 \mathrm{~mm}$ when depinning occurs. Similar scenarios are observed at all flow rates and in all injection configurations for which pinning takes place but the characteristic time scales may vary.

In the above experiments using a pure water flow, the transport of the fibers was only possible in the high aperture regions of the wedged fracture $F 2$. In the case of the polymer solution, the motion of the fiber is generally continuous in these regions; pinning only occurred in lower aperture parts of model $F 2$. Like for water, bumps appear on the fibers when they get pinned and they move toward its tip as they develop. Their amplitude and curvature 


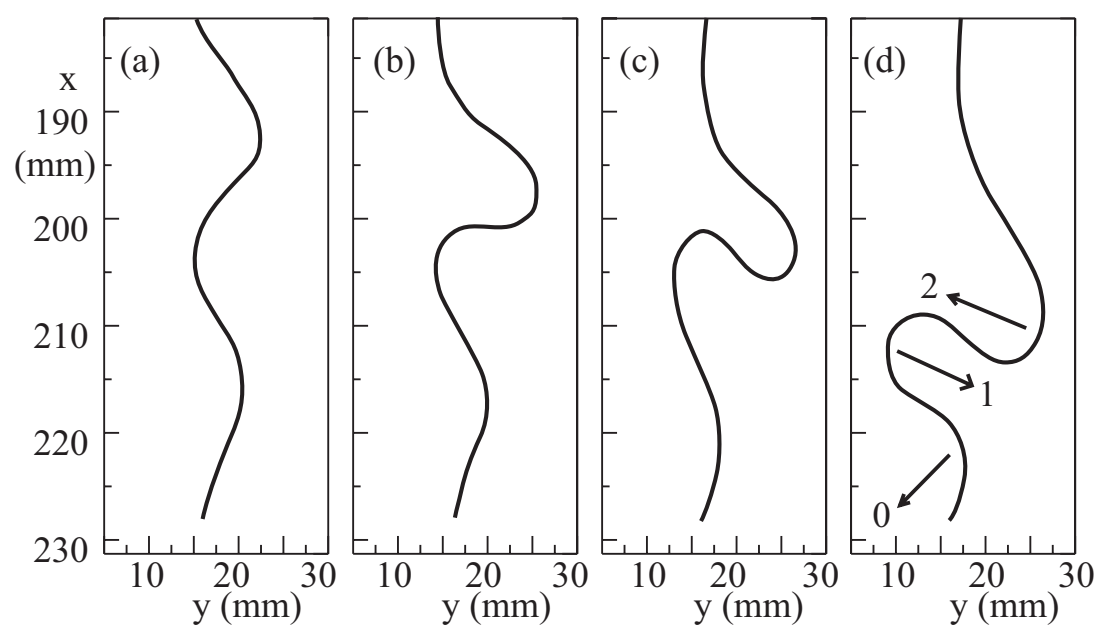

(e)

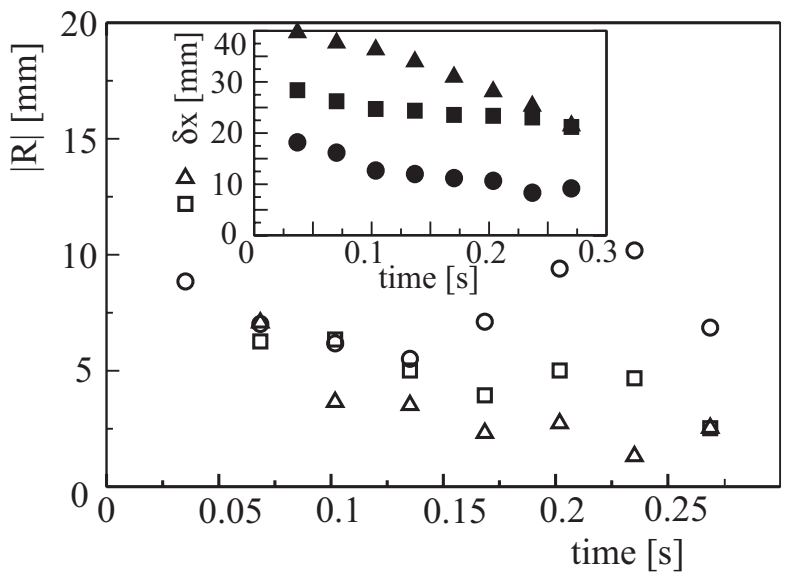

Fig. 8 (a,b,c,d) Fiber geometry at different times during a pinning event for a polymer flow $(U=230 \mathrm{~mm} / \mathrm{s})$ in the low aperture part of fracture F2. Leftmost (respectively rightmost) pictures: views of the fiber $0.1 \mathrm{~s}$ after pinning (respectively just before depinning). Points 0,1 and 2 and the corresponding symbols in the graph are defined as in Fig. 7. (e) Variation with time of the radius of the fiber at the 3 maximum curvature points designated by arrows in curve (d). Inset: time variation of the vertical distances $\delta x$ between these 3 points and the fiber tip.

are however significantly larger than for water. For this fluid which is characterized by an effective viscosity $\mu \sim 3 \mathrm{mPa}$.s, the elastic buckling calculation presented in the previous paragraph leads to a critical length $L \sim 5 \mathrm{~mm}$. The latter is closed to the value measured (see Fig.8c). Before depinning takes place: in Fig. 8a-d, for instance, an overhang appears as bump 1 is overtaken by 2 as shown by the two upper sets of points in the inset. Also, the variation of the radius of curvature with time is more complex than for the water flow and not always monotonous, which reflects the interaction between the different loops (Fig. 8e). The radii of curvature of loops 1 and 2 reach values of $\simeq 2 \mathrm{~mm}$ or even less: this is lower than the radius of loop 0 and much lower than the radii observed with water (see Fig. 7e). 
These larger deformations may result first from the occurence of pinning in narrower parts of the wedge than for water: at these locations, the depinning energies may therefore be expected to be higher. Also, the distances $\delta x$ vary faster with time in the case of the polymer solution (compare insets of Fig. 7d and Fig. 8e): in agreement with previous observations reported in Sec. 4.1, this suggests that the hydrodynamic forces experienced by the fiber are larger for these solutions.

\section{Irreversible pinning and fiber entanglement}
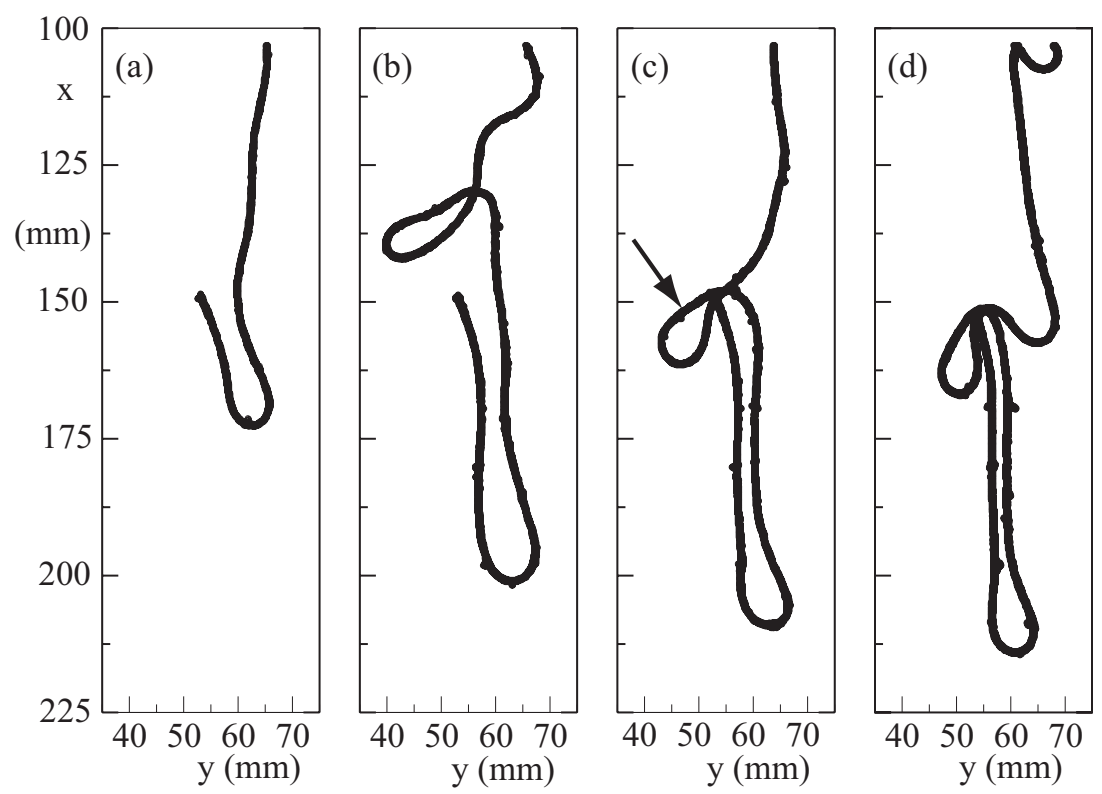

Fig. 9 Geometry of the fiber at different times during the build-up of a coil for the flow of a polymer solution in fracture $F 3$ (see Fig. 6b). Times elapsed after pinning are respectively $0.63,1.13,1.3$ and $1.8 \mathrm{~s}$. (from left to right)

In the previous section, pinning events of finite duration were discussed. Irreversible pinning is also frequent, for instance in the narrow parts of fracture $F 3$; the fiber generally becomes entangled around the pinning site and intertwined loops build up. Such a configuration is displayed in Fig. $6 \mathrm{~b}$ and corresponds to pinning in a narrow zone (yellow shade region near the top of the coil). At first, the fiber is deformed as in the case of transient pinning shown in Figs. 7 and 8. Then, bump 1 overtakes the pinned tip so that a loop appears at the right and moves downwards (Fig. 9a). Simultaneously, parts of the fiber located farther upstream move leftwards and build up a second, closed, loop (arrow in Fig. 9). This implies that, close to the pinning site, friction forces of the fiber with the walls are large enough to prevent the motion of the tip. Meanwhile, the parts of the fiber initially upstream from the tip are dragged downwards by the hydrodynamic forces and keep moving so that loops and deformations easily build-up. Finally, as the loops move down, they get locked on the pinning site and dangle on both sides (Fig. 9c). This further reduces the tension on 
the upstream fiber sections and new loops start to appear (Fig. 9d). The final interlocking of the fiber strands results in a strong blockage. The fiber cannot be released by increasing the velocity but only by pulling it upwards.

\section{Conclusion}

The present work has determined important features and requirements for the transport of flexible fibers through fractures. First, the transport of fibers by a flowing fluid in fractures with a small mean aperture and with very rough walls of self-affine geometry has been shown to be possible. However while, in smooth fractures, the friction of the fibers with the walls is negligible and does not influence their motion, its influence is much stronger for rough walls: this often results in pinning and blockage or in a jerky progression instead of a continuous one.

The aperture of the fracture (or, rather, its ratio to the fiber diameter) is, of course, a key factor but other geometrical parameters may be relevant. In the wedge-shaped rough model $F 2$, fiber transport is, as expected, easier in parts of the wedge where the mean aperture is large (typ. $\gtrsim 1 \mathrm{~mm}$ ); however, pinning may still occur in the more open regions. In the rough fracture $F 3$ with parallel walls, although of same roughness, the transport of the fiber is also easier in high permeability regions. However, the trajectory of the fibers does not coincide fully with the paths of highest aperture while pinning does not always take place at minimal aperture points. Other geometrical factors such as the local curvature of the walls and the spatial gradients of the aperture must be taken into account. Fiber transport is improved at higher flow velocities: stop-and-go motions may be turned into more continuous displacements at larger flow rates, although the velocity still decreases in the vicinity of the pinning sites.

Another key result is the enhancement of the mobility of the fibers when water is replaced by a polymer solution. Then, fibers can propagate along the full length of rough fractures of mean aperture only twice the fiber diameter; they also can access narrow regions where they would get stuck with water. At a same flow velocity, the motion of the fiber is faster and more continuous than for water; the effects of the friction with the walls are strongly reduced and the features of the propagation become comparable to those obtained for smooth walls. These results have important practical implications due to the widespread use of such solutions in many industrial processes. A first possible explanation is an increase of the drag forces due to the higher viscosity of these solutions, particularly at low shear rates; also, lubrication forces due to this higher viscosity may prevent the fibers from getting in contact with the walls and, therefore, reduce the friction. However, additional studies will be needed in order to understand whether the decreasing variation of the viscosity with the shear rate also plays a part in these results. Quantitative computations of the forces on fibers performed for Newtonian flows in smooth fractures will, in particular, have to be extended to shear-thinning fluids. The variation of the viscosity in the fracture gap may also influence the localization of the fiber inside it.

Finally, fibers often become deformed during their transit through rough fractures while they retain their shape in the smooth model. These deformations are particularly frequent and large during pinning events: then, upstream parts of the fibers are kept in motion by hydrodynamic forces while the tip is blocked. For transient pinning, the amplitude of the deformations of the fiber increases with time until the front tip is released and is larger for the polymer solution; then, the fiber recovers roughly its initial shape. For permanent 
pinning, the amplitude of the deformations increases further until loops appear and pile up on the pinning site, finally leading to entanglement.

The present experiments have determined several key characteristics of fiber transport in single fractures and of its dependence on the flexibility of the fibers, the characteristics of the fluid and the configuration of the aperture field. However, further work is needed to characterize more quantitatively the influence of these parameters. The velocity of fibers of different lengths in a water-flow has, for instance, been predicted for a smooth fracture but the case of rough fractures and/or shear thining flows remains open. Another important issue is the transfer of fibers from one fracture to another in a fracture network.

Acknowledgements We are indebted to R. Pidoux for the realization of the experimental setup. We are grateful to M. François, E.J. Hinch and J. Koplik for their enlightening comments. This work was supported by the Schlumberger Foundation in Paris and by Schlumberger-Doll Research in Boston through a fellowship and a research grant. We also benefited from funding by the EC through the STREP EGS PILOT PLANT (EC Contract SES-CT-2003-502706) and by the GdR 2990.

\section{References}

[Boffa et al. 1998] Boffa, J.M., Allain, C. and Hulin, J.P. Experimental analysis of fracture rugosity in granular and compact rocks. Eur. Phys. J. AP 2, 281-289 (1998).

[Boschan et al. 2007] Boschan, A., Auradou, H., Ippolito, I., Chertcoff, R. and Hulin, J.P. Miscible displacement fronts of shear thinning fluids inside rough fractures. Water Res. Res. 43, W03438 (2007).

[Bouard 1997] Bouard, R. Détermination de la traînée engendrée par un cylindre en translation pour des nombres de Reynolds intermédiaires. Z. angew. Math. Phys. 28, 584-596 (1997).

[Bouchaud 2003] Bouchaud, E. The morphology of fracture surfaces, a tool to understand crack propagation in complex materials. Surf. Rev. Lett. 10, 797-814 (2003).

[Butler and Shaqfeh 2002] Butler, J.E. and Shaqfeh, E.S.G.: Dynamic simulations of the inhomogeneous sedimentation of rigid fibres, J. Fluid Mech. 468, 205-237 (2002).

[Chiba et al. 1988] Chiba, K., Hayashi, S., Kojima, K. and Song, K-W.: fiber orientation in fiber suspension flow through a parallel plate channel and an abrupt contraction channel. J. Text. Mach. Soc. Japan, Transaction, 41, T19-T29 (1988)

[D'Angelo et al. 2007] D'Angelo, M.V., Auradou, H., Allain, C. and Hulin, J.P. Pore scale mixing and macroscopic solute dispersion regimes in polymer flows inside two-dimensional model networks. Phys. Fluids 19, 033103 (2007).

[D’Angelo et al. 2009] D’Angelo, M.V., Semin, B., Picard, G., Poitzsch, M., Hulin, J.P. and Auradou, H.: Flexible fiber transport by a fluid flow in fractures with smooth and rough walls, Proceedings of the $\mathrm{X}$ meeting on recent advances in the physics of fluids and their applications. Journal of Physics: Conference Series 166, 012001 (2009).

[Fan et al. 1998] Fan, X., Phan-Thien, N. and Zheng, R.: A direct simulation of fibre suspensions, J. NonNewtonian Fluid Mech. 74, 113-135 (1998).

[Forgacs and Mason 1959] Forgacs, O.L. and Mason, S.G.: Particle motions in sheared suspensions. X : Orbits of flexible threadlike particles. J. Coll. Int. Sci. 14, 473-491 (1959).

[Frei et al 2000] Frei, Ch. and Lüscher, P. and Wintermantel, E.:Thread-annular flow in vertical pipes. J. Fluid. Mech. 410, 185-210 (2000).

[Gentier et al. 1997] Gentier, S., Lamontagne, E. Archambault, G. and Riss, J. Anisotropy of flow in a fracture undergoing shear and its relationship to the direction of shearing and injection pressure. Int. J. Rock Mech. Min. Sci. 34, 412-412 (1997).

[Guyon et al. 2001] Guyon, E., Hulin, J.P., Petit, L. and Mitescu, C.: Physical Hydrodynamics, Oxford Press, Oxford, UK (2001).

[Habibi et al. 2007] Habibi, M., Ribe, N.M. and Bonn, D. Coiling of elastic ropes. Phys. Rev. Lett. 99, 154302 (2007).

[Herzhaft and Guazzelli 1999] Herzhaft, B. and Guazzelli, E.: Experimental study of the sedimentation of dilute and semidilute suspensions of fibres. J. Fluid Mech. 384, 133-158 (1999).

[Hinch 1976] Hinch, E.J.: The distortion of a ?exible inextensible thread in a shearing ?ow, J. Fluid Mech. 74, 317-333 (1976).

[Jeffery 1923] Jeffery, G.: The motion of ellipsoidal particles immersed in a viscous ?uid, Proc. Roy Soc. Ser. A 102, 161-179(1923). 
[Joung 2001] Joung, C.G., Phan-Thien, N., Fan, X.J.: Direct simulation of flexible fibers. J. Non-Newtonian Fluid Mech. 99, 1-36 (2001)

[Lagomarsino et al. 2004] Lagomarsino M.C.: biologically inspired problems concerning semi?exible Filaments. Ph.D. thesis, University of Leiden, (2004).

[Lagomarsino et al. 2005] Lagomarsino, M., Pagonabarraga, I. and Lowe, C.: Hydrodynamic induced deformation and orientation of a microscopic elastic filament. Phys. Rev. Lett. 94, 148104 (2005).

[Landau and Lifshitz 1986] Landau, L.D. and Lifshitz, E.M. Theory of Elasticity, Third Edition: Volume 7. Butterworth-Heinemann, Oxford, UK (1986).

[Leal 1975] Leal, L.G. The slow motion of slender rod-like particles in a second-order fluid. J. Fluid Mech. 69, 305-337 (1975).

[Llopis et al. 2008] Llopis, I., Lagomarsino , M.C., Pagonabarraga, I. and Lowe., C.P.: Cooperativity and hydrodynamic interactions in externally driven semiflexible filaments. Computer physics communications. 179, 150-154 (2008).

[Lowe 2003] Lowe, C.: Dynamics of filaments: modelling the dynamics of driven microfilaments. Phil. Trans. R. Soc. London B 358, 1543-1550 (2003).

[Moses 2001] Moses, K.B. Advani, S.G. and Reinhardt, A.: Investigation of fiber motion near solid boundaries in simple shear flow. Rheol Acta 40, 296-306 (2001).

[Ning and Melrose 1999] Ning, Z. and Melrose, J.R.: Numerical model for simulating mechanical behavior of flexible fibers. J. Chem. Phys. 111, 10717-10726 (1999).

[Poon et al. 1992] Poon, C., Sayles, R. and Jones, T.: Surface measurement and fractal characterization of naturally fractured rocks. J. Phys. D: Appl. Phys. 25, 1269-75 (1992).

[Purcell 1997] Purcell, E.M.: The efficiency of propulsion by a rotating flagellum. Proc. Natl . Acad. Sci . USA 94, 11307-11311 (1997).

[Richou et al 2005] A. Ben Richou, A., Ambari and M. Lebey and J. K. Naciri Drag force on a cylinder midway between two parallel plates at $\mathrm{Re}_{i i} 1$ Part2: moving uniformly (numerical and experimental). Chem. Eng. Sci. 60 2535-2543 (2005).

[Saintillan et al. 2005] Saintillan, D., Darve, E. and Shaqfeh, E. S. G.: A smooth particle-mesh Ewald algorithm for Stokes suspension simulations: the sedimentation of fibers. Phys. Fluids 17, 033301 (2005).

[Selker et al. 2006] Selker, J., van de Giesen, M., Westhoff, N., Luxemburg, W. and Parlange, M. B. Fiber optics opens window on stream dynamics. Geophys. Res. Lett. 33, L24401 (2006).

[Skjetne et al. 1997] Skjetne, P., Ross, R.F. and Klingenberg, D.J.: Simulation of single ?ber dynamics. J. Chem. Phys. 107, 2108-2121 (1997).

[Stockie 1998] Stockie, J. and Green, S.: Simulating the motion of flexible pulp fibres using the immersed boundary method. J. Comput. Phys. 147, 147-165 (1998).

[Taylor 1951] Taylor, G.I.: Analysis of the swimming of microscopic organisms. Proc. Roy. Soc. London A, 209, 447-461 (1951)

[Timoshenko and Gere 1962] Timoshenko, S.P. and Gere, J.M.: Theory of Elastic Stability. McGraw-Hill, New-York (1962).

[Tornberg and Shelley 2004] Tornberg, A.-K. and Shelley, M.J.: Simulating the dynamics and interactions of flexible fibers in Stokes flows. J. Comput. Phys. 196, 8-40 (2004).

[Yamamoto and Matsuoka 1993] Yamamoto, S. and Matsuoka, T.: A method for dynamic simulation of rigid and flexible fibers in a flow field, J. Chem. Phys. 98, 644-650 (1993).

[Yamamoto and Matsuoka 1995] Yamamoto, S. and Matsuoka, T.: Dynamic simulation of fiber suspensions in shear flow, J. Chem. Phys. 102, 2254-2260 (1995).

[Yasuda et al. 2002] Yasuda, K., Mori, N. and Nakamura, K.: A new visualization technique for short fibers in a slit flow of fiber suspensions. Int. J. Eng. Sci. 40, 1037-1052 (2002).

[Yeo et al. 1998] I. Yeo, W., Freitas, M. H. D. and Zimmerman, R. W. Effect of shear displacement on the aperture and permeability of a rock fracture. Int. J. Rock Mech. Min. Sci. 35, 1051-1070 (1998).

[Zimmerman et al. 2004] Zimmerman, W., Al-Yaarubi, A., Pain, C. and Grattoni, C. Non-linear regimes of fluid flow in rock fractures. Int. J. Rock Mech. Min. Sci. 41, 163-169 (2004).

[Zovatto et al 2001] Zovatto, L., Pedrizetti, G., Flow about a circular cylinder between parallel walls J. Fluid Mech. 440, 1305-1320 (2001). 